and 4/28 (14.3\%) referred for oxygen assessment. 33.3\% of eligible patients were referred to pulmonary rehabilitation. $77.5 \%$ required and were provided with a written educational pack, and a formal crisis management plan formulated for $49.3 \%$ cases. Inhaler technique was inadequate in $10.2 \%$ of patients and in part drove the prescribing changes.

Conclusion Using computer guiding consultation in real life practice resulted in substantial management recommendations and diagnostic revisions. COPD care can be improved, using computer guided consultation which enables non specialists to achieve it.

\section{Paediatric airway infections}

\section{S72 PAEDIATRIC PNEUMOCOCCAL EMPYEMA SEROTYPES HAVE NOT CHANGED FOLLOWING INTRODUCTION OF THE 13 VALENT PNEUMOCOCCAL VACCINE}

${ }^{1} \mathrm{MF}$ Thomas, ${ }^{2} \mathrm{C}$ Sheppard, ${ }^{3} \mathrm{M}$ Guiver, ${ }^{1} \mathrm{C}$ Simmister, ${ }^{4} \mathrm{MA}$ Elemraid, ${ }^{5} \mathrm{JE}$ Clark, ${ }^{6}$ SP Rushton, ${ }^{7}$ JY Paton, ${ }^{1}$ DA Spencer; ${ }^{1}$ Department of Respiratory Paediatrics, Great North Children's Hospital, Newcastle upon Tyne, United Kingdom; ${ }^{2}$ Center for Infections, Public Health England, London, United Kingdom; ${ }^{3}$ Molecular Diagnostic Services, Public Health England - North West, Manchester, United Kingdom; ${ }^{4}$ Department of Paediatrics, James Cook University Hospital, Middlesbrough, United Kingdom; ${ }^{5}$ Department of Paediatric Immunology and Infectious Diseases, Mater Hospital for Sick Children, Brisbane, United Kingdom; 'School of Biology, Newcastle University, Newcastle upon Tyne, United Kingdom; 'Department of Respiratory Medicine, Royal Hospital for Sick Children, Yorkhill, Glasgow, United Kingdom

\subsection{6/thoraxjn-2013-204457.79}

Introduction Pneumococcal infection is the leading cause of paediatric empyema in the UK. Prior to the change in the UK routine vaccination schedule from the seven valent conjugate pneumococcal vaccine (PCV-7) to the thirteen valent vaccine (PCV-13) in April 2010 four serotypes /serogroups-1, 3, 7A/F and $19 \mathrm{~A}$ accounted for $75 \%$ of culture negative pneumococcal empyema in UK children. Antigen for these four serotypes is not present in PCV-7 but is present in PCV-13. We examined the impact of PCV-13 on the incidence of disease due to serotypes $1,3,7 \mathrm{~A} / \mathrm{F}$ and $19 \mathrm{~A}$ using national surveillance data from the UK-ESPE study.

Methods Pleural fluid samples were forwarded from admitting hospitals. Those that were pneumococcal PCR positive underwent non-culture serotyping using a multiplex antigen detection assay capable of detecting 14 serotypes/groups $(1,3,4,5,6 \mathrm{~A} / \mathrm{C}$, $6 \mathrm{~B}, 7 \mathrm{~F} / \mathrm{A}, 8,9 \mathrm{~V}, 14,18,19 \mathrm{~A}, 19 \mathrm{~F}$ and $23 \mathrm{~F})$. Two time periods were analysed April 2008-April 2010 (PCV-7 era) and April 2010-April 2012 (PCV-13 era). Incidence rate ratios (IRR) were calculated for individual serotypes. Age distributions were compared by density plotting.

Results 380 samples (median age 3.8 years) were tested in the two time periods (191 PCV-7 era, 189 PCV-13 era). No reduction in the incidence of empyema caused by the four main serotypes/groups (IRR: Serotype 1-0.79 95\% CI (0.57-1.11), 3-0.91 (0.60-1.37), 7A/F-1.59 (0.85-3.04), 19A-2.42 (1.61-5.40)) was seen and 19A increased significantly. The age distribution of each serotype did not change between the two time periods.

Discussion The introduction of PCV-13 has not yet been associated with any reduction in the incidence of vaccine serotype pneumococcal empyema in children in the UK, in contrast to the changes following the introduction of PCV-7. The factors contributing to this remain unclear but may include a predominantly PCV-7 vaccinated cohort, insufficient herd immunity, inadequate immunological response to vaccine antigen or on-going secular trends. Continuing surveillance is essential and will provide important data on future trends to better understand these complex processes.

\section{S73 TRENDS IN EMPYEMA IN SCOTTISH CHILDREN 2000- 2011}

S Nath, SW Turner; University of Aberdeen, Aberdeen, UK

\subsection{6/thoraxjn-2013-204457.80}

Background An abrupt rise in empyema prevalence in children was noted in the UK and other countries during the late 1990s and early 2000s. Time trends in empyema prevalence in Scotland has not been described since 2005 at a time when prevalence appeared to be still rising ${ }^{1}$. A number of factors may have changed empyema prevalence since 2005 including the 2006 smoking ban and introduction of heptavalent (2006) and 13-valent (2010) pneumococcal vaccinations. Here we applied our previous methodology ${ }^{1}$ to test the hypothesis that the prevalence of childhood empyema continues to rise beyond 2005.

Methods This was a whole population study of 2000-2011 hospital admissions using ICD-10 diagnostic codes. As previously we captured admissions for pneumonia and croup to detect increasing prevalence of admissions with other respiratory presentations.

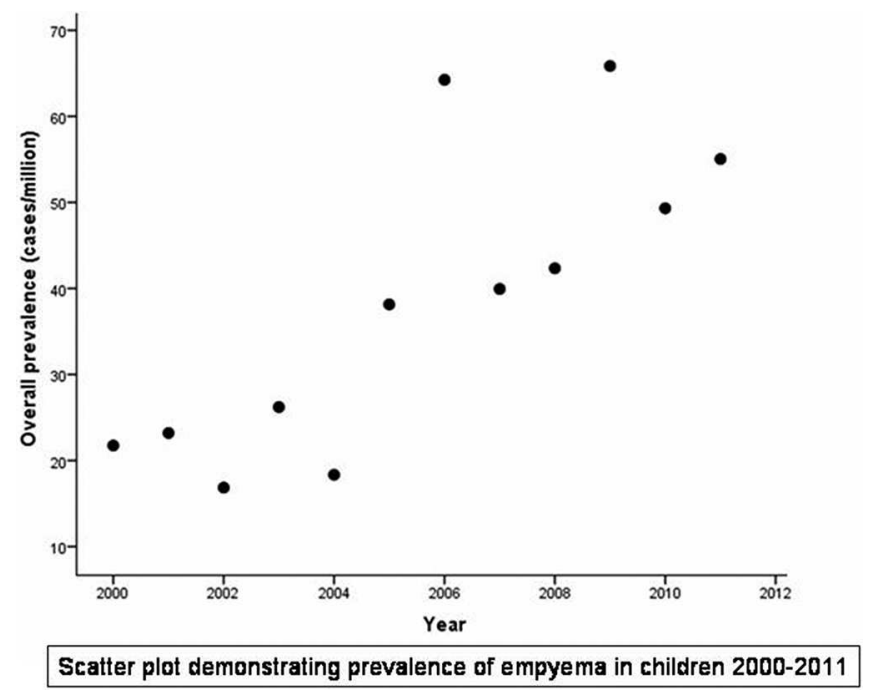

Abstract S73 Figure 1.

Results Over this 12 year period there were 398 cases of empyema. The prevalence rose from 22 cases/million in 2000 to 55 cases/million in 2011 (see figure), equivalent to a rise of 4 cases/million/year $\left(\mathrm{R}^{2}=0.81 \mathrm{p}=0.002\right)$. Within the $1-4$ year age range, empyema prevalence rose by 10 cases/million/year $\left(\mathrm{R}^{2}=0.86 \mathrm{p}<0.001\right)$ whilst prevalence did not change for the under 1 and 10-14 year old age range. The prevalence of croup and pneumonia for did not change during 2000-2011 suggesting that increased empyema prevalence did not reflect increasing respiratory admissions or increasing pneumonia prevalence.

Conclusion The prevalence of empyema in Scottish children has continued to rise beyond 2005 and the reason for this is not clear. Public health initiatives introduced since 2005 do not appear to have altered empyema prevalence in children. 


\section{REFERENCES}

1. Roxburgh cs et al. Trends in pneumonia and empyema in Scottish children in the past 25 years. Arch Dis Child 2008;93:316-8.

\section{S74 IS BRONCHOSCOPY NEEDED IN CHILDREN WITH PERSISTENT BACTERIAL BRONCHITIS?}

RK Narang, K Bakewell, J Peach, S Clayton, M Samuels, J Alexander, W Lenney, FJ Gilchrist; Academic Department of Child Health, University Hospital of North Staffordshire, Newcastle Road, Stoke-on-Trent, United Kingdom

\subsection{6/thoraxjnl-2013-204457.81}

Introduction and Objectives Persistent bacterial bronchitis (PBB) is increasingly recognised as a cause of chronic cough in young children but there is lack of consensus about investigation and treatment. At UHNS, children with a wet cough for $>6$ weeks unresponsive to oral antibiotics prescribed by the GP are investigated with CXR, baseline immune function and flexible bronchoscopy with bronchoalveolar lavage (FB-BAL). Patients with confirmed PBB are then treated with a prolonged course of an appropriate antibiotic. Some centres reserve FB-BAL for those who do not respond to blind treatment with co-amoxiclav or clinically relapse. The objective was to review bronchoscopic findings and immune function in children with chronic cough to determine which investigations are necessary.

Methods A retrospective case note review of all children investigated for chronic cough between May 2011 and June 2013.

Results The notes of 44 children with chronic cough were reviewed. BAL samples were taken from 6 lobes in every patient. Median (IQR) age at bronchoscopy was $3.3(1.8-4.4)$ years. Positive BAL cultures were obtained from 35 patients $(80 \%)$. Ten patients $(23 \%)$ isolated $\geq 2$ organisms. Haemophilus influenza was identified in 20 (46\%), Moraxella catarrhalis in 11 (25\%), Staphylococcusl aureus in 10 (23\%) and Streptococcus pneumoniae in 6 (14\%). Candida albicans, Group A Streptococcus, Haemophilus parainfluenzae and a gram negative bacillus were each identified in 1 patient (2\%). In $13(30 \%)$ at least 1 organism was isolated that was unlikely to respond to co-amoxiclav. If the right middle lobe (RML) had been the only lobe sampled (as per ERS guidance) organisms would have been missed in 14 patients (32\%). Suboptimal functional antibodies to Haemophilus influenza or Pneumococcus were identified in 7 patients (16\%). Appropriate antibiotics were prescribed for all patients with a positive culture. Co-amoxiclav was the most commonly prescribed antibiotic and was used in 20 patients (57\%). Treatment duration varied between 4 and 8 weeks.

Conclusions FB-BAL is a useful investigation to aid the diagnosis and guide treatment in PBB. The best time to perform FB-BAL is not known. In PBB a number of organisms will be missed if BAL is only taken from the RML.

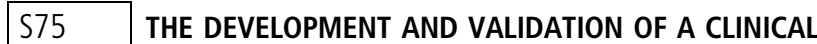 SEVERITY SCORE FOR INFANTS WITH BRONCHIOLITIS}

${ }^{1} \mathrm{C}$ van Miert, ${ }^{2} \mathrm{~J}$ Abbott, ${ }^{1} \mathrm{~F}$ Verhoeff, ${ }^{3} \mathrm{~S}$ Lane, ${ }^{2} \mathrm{~B}$ Carter, ${ }^{3} \mathrm{PS}$ McNamara; ${ }^{1}$ Alder Hey Children's NHS Foundation Trust, Liverpool, UK; ${ }^{2}$ University of Central Lancashire, Preston, UK; ${ }^{3}$ University of Liverpool, Liverpool, UK

\subsection{6/thoraxjn-2013-204457.82}

Introduction and Objectives Bronchiolitis is a viral lower respiratory tract infection of infancy. ${ }^{1} 1-3 \%$ of all infants are admitted to hospital with $3 \%$ of hospitalised infants requiring critical care. $^{2}$

Objective To develop and validate a scoring instrument for use by health care professionals (HCPs) in infants with bronchiolitis which has clinical utility.

Methods Psychometric methods were used to develop the scoring instrument and to test the instrument for validity and reliability in a variety of clinical locations.

Results Item generation, reduction \& instrument development: 101 items were identified from the literature and focus group workshops (families \& HCPs). Consensus for importance was achieved for 45 items (Table 1) following a Delphi survey of 195 HCPs. A scoring instrument with 12 domains was developed.

\begin{tabular}{|c|c|c|c|}
\hline Respiratory Symptoms & Risk Factors \& Miscellaneous symptoms & Level of Consciousness & Hydration \& Perfusion \\
\hline 1. Respiratory rate & 21. Day of illness & 31. Alertness & 37. Feeding \\
\hline 2. Grunting & 22. Personal concerns / 'gut' feeling & 32. Irritability & 38. Urine output \\
\hline 3. Nasal flare & 23. Parental concerns & 33. Drowsiness & 39. Central capillary refill time measured \\
\hline 4. Recession & 24. General condition & 34. Responds to pain & over a given time \\
\hline 5. Accessory muscle use & 25. Chronic lung disease & 35. Unresponsive & 40. Peripheral perfusion \\
\hline 6. Dyspnoea & 26. Congenital heart disease & 36. AVPU scale (alert, verbal, & 41. Mottled appearance \\
\hline 7. Tracheal tug & 27. HIV/ immunodeficiency & pain, unresponsive) & 42. Sunken eyes \\
\hline 8. Respiratory pattern & 28. Gestational age ( $<37$ weeks) & & 43. Sunken fonatanelle \\
\hline 9. $\mathrm{PaCO}_{2}$ on blood gas analysis & 29. Low birth weight & & 44. Heart rate \\
\hline 10. Ph on blood gas analysis & 30. Bacterial or viral co-infection & & 45. Pallor \\
\hline 11. Apnoea & & & \\
\hline 12. Stridor & & & \\
\hline 13. Head bobbing & & & \\
\hline 14. Using stomach to breathe & & & \\
\hline 15. Cyanosis & & & \\
\hline 16. Effort of breathing & & & \\
\hline 17. Air entry & & & \\
\hline 18. Oxygen requirements & & & \\
\hline 19. Oxygen saturation & & & \\
\hline 20. See saw chest motion & & & \\
\hline
\end{tabular}

